\title{
Changes in the corneal thickness and limbus after 1 year of scleral contact lens use
}

\author{
Beatriz de Luis Eguileor $\mathbb{D}^{1} \cdot$ Arantxa Acera $^{2}$ - Alaitz Santamaría Carro ${ }^{1} \cdot$ Raquel Feijoo Lera ${ }^{1} \cdot$ \\ Julene Escudero Argaluza ${ }^{3}$. Jaime Etxebarria Ecenarro ${ }^{1}$
}

Received: 16 January 2019 / Revised: 16 October 2019 / Accepted: 21 November 2019 / Published online: 10 December 2019

(c) The Author(s), under exclusive licence to The Royal College of Ophthalmologists 2019

\begin{abstract}
Purpose To assess the physiological changes in the cornea over time in patients with irregular cornea fitted with Rose K2 XL gas-permeable scleral contact lenses.

Methods Prospective study of 16 eyes of patients who did not tolerate gas-permeable corneal contact lenses and were fitted with Rose K2 XL scleral lenses. We assessed the central vault and the corneal thickness centrally and at peripheral regions (2 to $5 \mathrm{~mm}$ annulus). All these measures were obtained by anterior segment optical coherence tomography. The measurements were taken immediately after fitting the lenses and 1,6 and 12 months later. Prior to the study and at 1 year, we performed an objective test for diagnosing limbal stem cell deficiency (Limbokit).

Results The mean vault was $201.7 \pm 82.3 \mu \mathrm{m} 20 \mathrm{~min}$ after fitting the contact lens; $189.4 \pm 94.0 \mu \mathrm{m}$ at 1 month; $165.1 \pm$ $75.9 \mu \mathrm{m}$ at 6 months and $142.1 \pm 76.8 \mu \mathrm{m}$ at 1 year, the values at 6 and 12 months being significantly different to baseline. After 1 year, the central corneal thickness had increased by $2.3 \%(\mathrm{IQR}=5.6)$, but the changes were only significant for the superior thickness. There is no limbal stem cell deficiency after 1 year of scleral contact lens use.

Conclusions After use of Rose K2 XL scleral contact lenses, the corneal physiology of patients with an irregular cornea remains unchanged, as assessed by corneal thickness measurements and the Limbokit test. In all cases, however, the vault decreased over time.
\end{abstract}

\section{Introduction}

The development of new laboratory diagnostic techniques and tools has enabled us to carry out a more accurate assessment of the fitting of scleral contact lenses. At the same time, with improvements in materials and designs, there has been a growth in their use [1].

The therapeutic usefulness of scleral contact lenses both for irregular corneas and ocular surface

Supplementary information The online version of this article (https:// doi.org/10.1038/s41433-019-0729-z) contains supplementary material, which is available to authorized users.

\footnotetext{
Beatriz de Luis Eguileor

beatrizdeluis@gmail.com

1 Hospital Universitario de Cruces, Barakaldo, Spain

2 Bioftalmik, Parque Tecnológico de Vizcaya, Derio, Spain

3 Unidad de Epidemiología Clínica, Hospital Universitario Cruces/ Biocruces Health Research Institute, Barakaldo, Spain
}

diseases is supported by numerous studies conducted over the last 8 years [2-14]. In contrast, little is still known about the relationship between scleral contact lens and the cornea, the optimum fitting values are still not perfectly established [14]. Some mathematical models have been developed describing the ideal lens characteristics to ensure that the relationship between scleral contact lens and the cornea does not affect the physiology of the cornea, and authors have concluded that it is necessary to use contact lenses with a high oxygen permeability ( $\mathrm{Dk}>125$ ), lens thickness of $200-250 \mu \mathrm{m}$ and a vault no higher than $200 \mu \mathrm{m}$ [15-17]. Therefore, the distance between the lens and the cornea being too small would compromise corneal mechanics, while the distance being too large could limit the delivery of oxygen to the cornea.

Studies of the impact of scleral contact lenses in corneal thickness have reported an increase in corneal thickness of around $2 \%[18,19]$. Smith et al. [20] indicated that use of a scleral contact lens overnight induces corneal swelling of 4.9 to $17.5 \%$. 
Most studies reviewed, both those analysing the relationship between scleral contact lenses and cornea and those assessing changes in corneal thickness, have been carried out in healthy individuals and over short periods of time. One exception is the study of corneas in patients with keratoconus who use scleral contact lenses by Soeters et al. [18]. These authors performed pachymetry immediately and 1 week after scleral contact lens removal and reported a $2.5 \%$ increase in corneal thickness.

The vault observed varies over time after fitting scleral contact lenses [21], as the conjunctival region where the lens rests is spongy and it progressively "settles" into the tissue.

Furthermore, the corneal limbus has a key role in the regeneration of the corneal epithelium, since it contains the stem cells responsible for the regeneration of this tissue. Limbal stem cell deficiency is clinically characterised by a vascularisation and invasion of conjunctival cells onto the corneal epithelium, chronic inflammation and persistent and recurrent epithelial defects, photophobia and loss of vision. The diagnosis of severe limbal stem cell deficiency is straightforward, especially if there is history of trauma or an underlying hereditary condition, but mild and moderate cases may go undetected.

It is, however, possible to detect such cases of limbal stem cell deficiency, even if they are subclinical, by impression cytology or fluorophotometry [22]. Impression cytology involves the collection of several layers of corneal and conjunctival epithelium and analysis of this tissue through one of various techniques (PAS-haematoxylin staining, immunofluorescence among others), allowing us to study the most superficial cell layers of both types of epithelia [23]. It is a minimally-invasive technique that is easy to perform and can be repeated over time. Further, a molecular test is commercially available for detecting limbal stem cell deficiency, namely, Limbokit (Operon S.A, Zaragoza, Spain). This test has higher specificity and sensitivity than impression cytology with PAS-haematoxylin staining $[24,25]$.

After reviewing the data available, we observed a gap in the literature concerning the long-term behaviour of scleral contact lenses in patients with irregular corneas. To help to fill this gap, the purpose of our study was to assess changes in the corneal thickness and limbus over time in patients with irregular corneas fitted with the Rose $\mathrm{K} 2 \mathrm{XL}^{\mathrm{TM}}$ scleral contact lenses.

\section{Material and methods}

\section{Patients}

This prospective study was approved by the Clinical Research Ethics Committee of Cruces University Hospital.
In accordance with the Declaration of Helsinki, participants were provided with information regarding the study and asked to give written informed consent.

In this study, we included patients seeking medical attention in the Ocular Surface Unit of the Ophthalmology Service at Cruces University Hospital, because they were only able to tolerate gas-permeable corneal contact lenses for $4 \mathrm{~h}$ or less or were completely unable to tolerate such lenses. We assessed 16 eyes (from 9 patients): $56.3 \%$ of the eyes where right eyes, the sex ratio was $1: 1$, and a mean age was $37.6 \pm 12.0$ years. The causes of irregular cornea were: keratoconus in $37.5 \%$ of cases, keratoconus with intrastromal corneal ring segments in $37.5 \%$, deep anterior lamellar keratoplasty in $18.8 \%$ and penetrating keratoplasty in $6.2 \%$.

Prior to fitting each scleral contact lens, we carried out a comprehensive ophthalmological assessment including: uncorrected and corrected visual acuity (VA) measured with a Snellen chart; examination of the anterior and posterior segments; corneal topography (Pentacam, Oculus, Wetzlar, Germany); anterior segment optical coherence tomography with a RTVue-100 system (Optovue Inc, Fremont, California, USA) to assess corneal thickness and Goldmann applanation tonometry. The limbal epithelium was tested using a commercial kit (Limbokit) which assessed the existence or nonexistence of limbal stem cell deficiency. This kit contains strips that detect the MUC5AC transcript in the corneal epithelium indicating limbal stem cell deficiency.

Patients were required to obtain a negative result in the Limbokit test before being fitted with the scleral contact lenses and tolerate wearing these lenses at least $8 \mathrm{~h}$ per day before inclusion in the study. We screened 17 eyes, only one of them had positive Limbokit. We analysed the changes observed over a 1 year period of wearing this type of contact lenses. In addition, we used the Limbokit test that provides an objective measure of limbal stem cell deficiency, to determine whether the corneal limbus is affected by wearing scleral contact lenses.

\section{Contact lenses}

The contact lenses used, Rose K2 XL, are made of tisifilcon A (Menicon Z, Menicon Co. Ltd., Nagoya, Japan), and have an aspheric back optical surface, the optical zone decreases as the base curve steepens.

In all eyes studied, we used standard $14.60 \mathrm{~mm}$ diameter lenses. The base curve of the first trial lens was selected following the manufacturer's recommendations, taking into account keratometry $(\mathrm{K})$ and horizontal visible iris diameter measurements.

The selected trial lens was filled with preservative-free sterile saline solution and sodium fluorescein (Haag-Streeit, 
Koeniz, Switzerland), and was then inserted into the patient's eye. Immediately after insertion, the central fit (central cornea vault) was evaluated and the trial contact lens was changed where necessary. A progressively steeper or flatter base curve was selected until an adequate vault was achieved, that is, a central vault of $100-300 \mu \mathrm{m}$.

After that, the edge lift was evaluated and adjustments made until achieving an optimum peripheral fit: a peripheral band $\sim 0.8-1 \mathrm{~mm}$ wide, no blanching of the conjunctival vessels and no hyperaemia to conjunctival vessels. The contact lens should extend 1.3-1.5 mm outside the limbus. If the fit seemed appropriate, the lens was re-evaluated after $20 \mathrm{~min}$. Having allowed this time for the lens to settle, sodium fluorescein was re-applied to check the fit. No apex staining or blanching of the conjunctival vessel was considered acceptable. We also evaluated the fit with anterior segment OCT (Fig. 1). With the set of trial contact lenses available, the base curve of the contact lens could be changed as necessary to achieve an adequate central fit. In contrast, as the trial set only had lenses with standard lifts, the changes required in the edge lift were empirical. Overrefraction was performed with the selected contact lens. Then, the lens chosen was ordered from the laboratory.

The contact lens received was fitted and evaluated with the slit lamp and OCT to determine whether the fit was good; if not, a different lens was ordered and the process was repeated until a good fit was achieved. If it was confirmed to be a good fit, the patient was taught how to insert, remove and clean the lens. Then, the patient was instructed to increase lens wearing time by $1 \mathrm{~h}$ a day and the contact lens fit was evaluated 1 week, 1 month, 6 months and 1 year later.

Moreover, the comfortable wearing time was evaluated at 1 month, 6 months and 1 year later. In the different visits, the patients were asked about the number of hours that they can use the scleral contact lenses without problems.

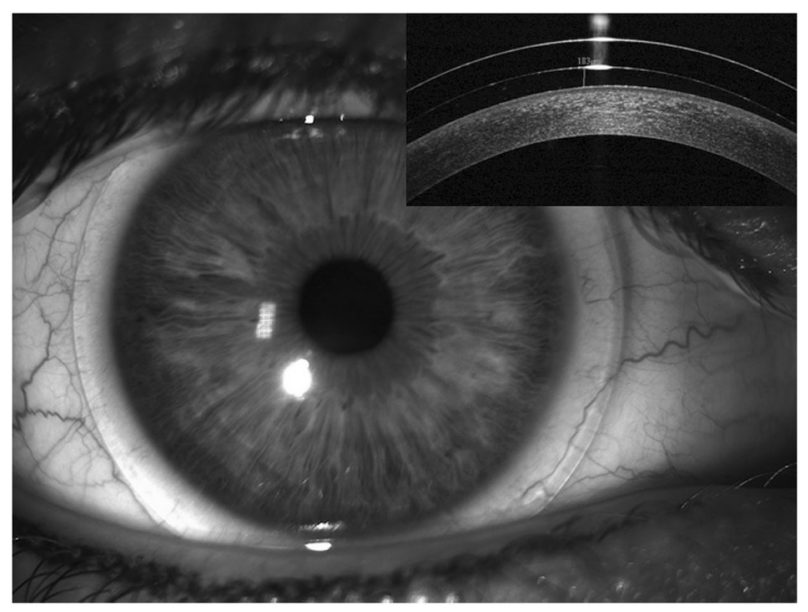

Fig. 1 Fit of the Rose K2 XL contact lens.

\section{Anterior segment optical coherence tomography}

OCT enables us to obtain high-resolution images using a Michelson interferometer and a low-coherence light source. For the measurements we required, the RTVue-100 system uses a corneal module that operates at a wavelength of $830 \mathrm{~nm}$, providing a scan depth of up to $8 \mathrm{~mm}$ with an axial resolution of $10-15 \mu \mathrm{m}$.

With RTVue-100 OCT, the mean repeatability of corneal thickness measurements is $1.2 \pm 0.8 \mu \mathrm{m}$ [26-28] in normal corneas and 5.2 $\pm 5.6 \mu \mathrm{m}$ [29] in keratoconus. To assess corneal oedema, we measured corneal thickness with the RTVue-100 system (Fig. 2) before, and 1, 6 and 12 months after fitting the contact lenses.

The scan pattern to determinate the pachymetry consisted of eight high-definition meridional scans. The corneal thickness profile was measured by an automated algorithm by interpolating the thickness profiles on the eight meridians. The pachymetry map was divides into zones by octants and annular rings $(2,5$ and $6 \mathrm{~mm})$. The average corneal thickness of each zone was displayed in a sector map.

Anterior segment OCT was also used to obtain measurements of the central thickness of the fitted contact lenses and changes in the central clearance (vault) over time. For this, images had to be taken horizontally, without the patient blinking and with no artefacts, and we needed to see a bright line from the reflex saturation beam running through the centre of the image. Furthermore, the line of the measurement had to cross always the same crypt of the iris. We performed three consecutive measurements, by the same examiner, in each eye to verify the repeatability. All the measurements were taken around the same time between 10:00 am and 2:00 pm, with patients using the contact lenses every day for at least $8 \mathrm{~h}$. That is, for the corneal thickness measurements, patients kept using the contact lenses every day (with no rest days) and these measurements were taken immediately after removing the lenses.

\section{Limbokit}

The Limbokit is an easy-to-use test, with a high sensitivity and specificity for the molecular diagnosis of limbal stem cell deficiency. It is based on the detection of MUC5AC transcripts amplified and immobilised on a test strip. Specific detection of the cDNA obtained by reverse transcription of MUC5AC mRNA from corneal epithelial samples confirms the presence of goblet cells, and hence, the diagnosis of limbal stem cell deficiency.

The test is performed by placing a membrane from the kit on the patient's cornea under topical anaesthesia. Corneal surface cells adhere to the membrane by corneal impression cytology. For this study, patient samples collected in this 


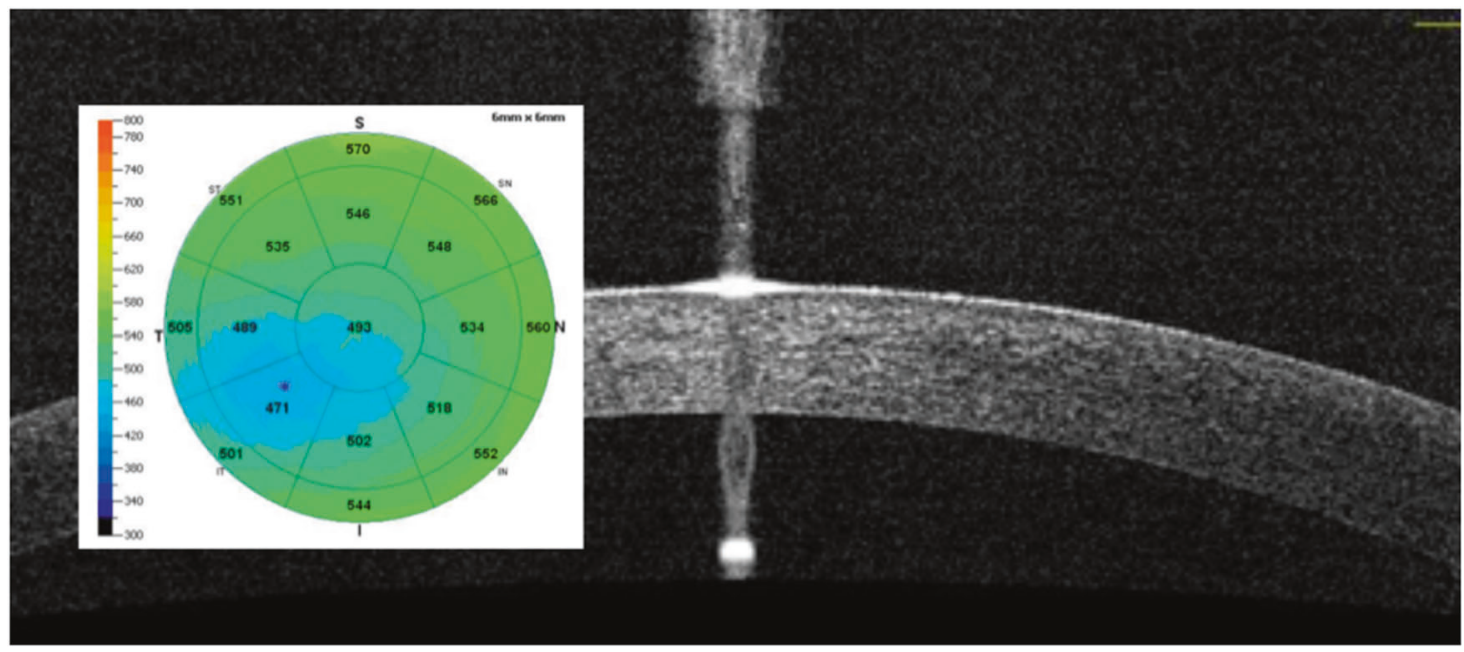

Fig. 2 Measurement of corneal thickness with RTVue-100 optical coherence tomography.

way were sent to the laboratory for processing and subsequent analysis. RNA was extracted from these samples and subsequently cDNA reverse transcription was performed and the results analysed.

\section{RNA extraction from corneal epithelium}

The RNA from the corneal cells adhered to the membrane was extracted using an extraction kit (RNeasy Plus Micro kit, Qiagen). First, the membrane was placed into $350 \mu \mathrm{l}$ of Buffer RLT Plus provided in the extraction kit to facilitate the separation of the cells under centrifugation and the mixture was centrifuged, triggering the release of the cell membranes and enabling us to extract the genetic material together with the cell organelles. After sample homogenisation, genomic DNA and organelle RNA of interest for our study were separated using specific columns included in the kit. Once the RNA had adhered to the column filter, it was diluted in $16 \mu \mathrm{l}$ of sterile water for cDNA reverse transcription.

\section{Reverse transcription of RNA to CDNA}

Reverse transcription was performed with a specific kit (Transcriptor First Strand cDNA Synthesis Kit; Roche Diagnostics, Mannheim, Germany). For this, we followed the protocol of the test manufacturer (Limbokit, Operon S.A, Zaragoza, Spain), and collected a total of $20 \mu \mathrm{l}$ of cDNA.

\section{Polymerase chain reaction (PCR) and detection}

Using $10 \mu \mathrm{l}$ of the sample obtained, the cDNA was amplified by PCR, following the specific protocol indicated by the test manufacturer (Limbokit). Fragments of cDNAs from MUC5AC (the study gene) and GAPDH (a housekeeping gene, used as an internal control) were amplified simultaneously.

During the PCR analysis, the amplified DNA samples were labelled with biotin. DNA probes that specifically recognise the amplified sequences during PCR are covalently bound to the test membranes. For each of the genes, there is a probe that will detect the amplification. The strips also have a control probe to monitor the reaction and coloured lines to indicate the position of the strip and help with the interpretation of results (Supplementary Fig. 1).

\section{Statistical analysis}

For the descriptive analysis, categorical variables were described using frequencies and percentages, and quantitative variables using mean, standard deviation, minimums and maximums or median and interquartile range. The nonparametric Friedman test for related samples was used to compare the results over time, setting the global level of significance at $p<0.05$. When we found overall significance, the Wilcoxon test for related samples was used, applying the Bonferroni correction. Pearson's correlation coefficient was used to assess the relationship between quantitative variables. Statistical analysis was carried out using IBM SPSS Statistics for Windows, Version 23.0.

\section{Results}

For the corneas of the eyes analysed, the mean horizontal visible iris diameter was $11.07 \pm 0.2 \mathrm{~mm}(11.5-12.0)$ and the mean keratometry reading was $7.1 \pm 0.3 \mathrm{~mm}(6.6-7.7)$. The Rose K2 XL contact lenses fitted had a mean radius of $6.8 \pm 0.2 \mathrm{~mm}$ (6.4-7.2), edge lift of $0.2 \pm 0.8$ [(-1.5) $-(1.0)]$ and optical power of $-7.3 \pm 3.1$ dioptres $[(-13.0)-(-1.5)]$. 
Table 1 Visual acuity and comfortable wearing time.

\begin{tabular}{|c|c|c|c|c|c|}
\hline & \multirow{2}{*}{$\begin{array}{l}\text { Corneal contact lenses } \\
\text { Pre-study }\end{array}$} & \multicolumn{4}{|c|}{ Rose K2 XL contact lenses } \\
\hline & & Recently fitted & 1 month & 6 months & 12 months \\
\hline Visual acuity (logMAR) & $0.3 \pm 0.2(0.1-0.7)$ & $0.1 \pm 0.1(0.0-0.2)$ & $0.1 \pm 0.1(0.0-0.2)$ & $0.1 \pm 0.1(0.0-0.2)$ & $0.1 \pm 0.1(0.0-0.2)$ \\
\hline $\begin{array}{l}\text { Comfortable wearing time } \\
\text { (hours) }\end{array}$ & $\leq 4$ & - & $9.5 \pm 3.0(6.0-14.0)$ & $11.3 \pm 2.4(8.0-14.0)$ & $12.0 \pm 2.4(8.0-16.0)$ \\
\hline
\end{tabular}

Table 2 Corneal thickness $(\mu \mathrm{m})$ over time. Wearing time.

\begin{tabular}{llllll}
\hline & $\begin{array}{l}\text { Central } \\
p=0.306\end{array}$ & $\begin{array}{l}\text { Superior } \\
p=0.003\end{array}$ & $\begin{array}{l}\text { Inferior } \\
p=0.700\end{array}$ & $\begin{array}{l}\text { Nasal } \\
p=0.497\end{array}$ & $\begin{array}{l}\text { Temporal } \\
p=0.339\end{array}$ \\
\hline Pre-fitting & $489.8 \pm 52.4(423-620)$ & $562.2 \pm 39.2(502-625)$ & $517.5 \pm 65.3(397-623)$ & $559.4 \pm 59.8(507-741)$ & $511.1 \pm 36.6(447-584)$ \\
1 month & $496.1 \pm 57.5(423-649)$ & $573.6 \pm 44.4(510-666)$ & $522.1 \pm 70.4(401-650)$ & $552.5 \pm 46.1(489-628)$ & $517.2 \pm 40.8(452-602)$ \\
6 months & $497.9 \pm 62.7(422-656)$ & $572.3 \pm 40.8(505-649)$ & $510.4 \pm 66.7(405-663)$ & $558.4 \pm 42.7(492-636)$ & $515.2 \pm 45.2(455-620)$ \\
12 months & $500.9 \pm 59.4(420-656)$ & $579.7 \pm 35.0(522-642)$ & $515.3 \pm 66.3(404-655)$ & $567.6 \pm 44.1(500-636)$ & $512.2 \pm 43.8(450-620)$ \\
\hline
\end{tabular}

Corneal thickness: centrally (central $2 \mathrm{~mm}$ ); and at locations temporal, nasal, superior and inferior (from 2 to $5 \mathrm{~mm}$ from the centre)

The thickness of the contact lenses, as measured with RTVue-100 OCT, was 207.9 $\pm 63.3 \mu \mathrm{m}$ (145-332).

Prior to fitting the Rose K2 XL contact lenses, the bestcorrected $\log$ MAR VA with the gas-permeable corneal contact lenses was $0.3 \pm 0.2(0.7-0.1)$. After the final fitting of the Rose K2 XL contact lenses, VA was assessed at each of the visits, the logMAR VA reaching $0.1 \pm 0.1(0.2-0.0)$ at 1 year. The change in VA over the follow-up was not statistically significant $(p=0.552)$, but the difference was significant comparing with the VA obtained with the corneal contact lenses that they used before the study $(p<$ 0.01 ). The mean comfortable wearing time of the Rose K2 XL contact lenses after 1 year of use was $12.0 \pm 2.4(8-16) \mathrm{h}$ a day (Table 1).

In corneal pachymetry with anterior segment OCT, we observed median increases in the central corneal thickness of $2.3 \%(\mathrm{IQR}=5.6)$ and in superior, inferior and nasal corneal thicknesses of $2.4 \%(\mathrm{IQR}=4.9), 0.0 \%(\mathrm{IQR}=9.0)$ and $2.1 \%$ $(\mathrm{IQR}=5.1)$, respectively, while the temporal corneal thickness showed a median decrease of $0.8 \%(\mathrm{IQR}=7.3)$. These changes in corneal thickness over time were only statistically significant in the case of the measurement superior to the corneal vertex $(p=0.003)$ (Table 2).

Analysing the contact lens-to-cornea distance, that is, the vault, we found a statistically significant decrease after 1 year of use of the Rose $\mathrm{K} 2 \mathrm{XL}^{\mathrm{TM}}$ contact lenses, of $29 \pm$ $25 \%$ with respect to the initial value (Table 3 ).

In Pearson's correlation analysis, we did not find a significant relationship between changes in corneal thickness over the year of use of the contact lenses and the central thickness of the lenses fitted $(r=0.196 ; p=0.467)$. Similarly, we did not find a significant relationship between the changes in vault and in corneal thickness over time $(r=$ $-0.221 ; p=0.411)$. The Limbokit test carried out after 1
Table 3 Vault $(\mu \mathrm{m})$ over time.

\begin{tabular}{lll}
\hline Day 1, day of fitting & $202 \pm 82(107-374)$ & \\
1 month & $189 \pm 94(78-396)$ & $p=0.333$ \\
6 months & $165 \pm 75(70-300)$ & $p=0.013$ \\
12 months & $142 \pm 77(50-315)$ & $p=0.002^{*}$ \\
\hline
\end{tabular}

*Statistically significant after Bonferroni correction $(p=0.0083)$

year of use of the Rose K2 XL contact lenses was negative in all the eyes analysed.

\section{Discussion}

In the last decade, several studies have found that VA improves when scleral contact lenses are fitted to patients with irregular corneas $[2-4,8,30]$. In this study, we found that VA with Rose $\mathrm{K} 2 \mathrm{XL}^{\mathrm{TM}}$ improved in all patients, being at least 2 lines better than the best-corrected VA with their corneal contact lenses, and the improvement was maintained throughout the year of follow-up.

Daily wearing time can be considered a good indicator of tolerance of contact lenses and vision correction success with scleral lenses. The mean wearing time in our patients was $12.0 \pm 2.4 \mathrm{~h}$, which may be relevant at the time of interpreting any changes in corneal thickness. Few studies have reported data on this in patients with irregular corneas and, those that do, report a mean wearing time of only $8 \mathrm{~h}$ $[3,31,32]$.

To our knowledge, however, ours is the first study to describe objective changes in the cornea after scleral contact lens use, and to report changes in corneal thickness as an indicator of corneal oedema throughout a year of follow-up. 
Any process that affects the supply of oxygen to the cornea might alter corneal physiology, corneal oedema being one of the most common complications. To avoid this, we must ensure a sufficient supply of oxygen to the cornea, normal tear osmolarity and a lack of mechanical and/or chemical irritation. To assess the level of oedema, we can quantify the percentage change in corneal thickness with respect to baseline, there being an association between corneal swelling and changes in corneal thickness [33]. There is a physiological phenomenon of morning oedema caused by eyelid closure during the night. Specifically, eyelid closure during sleep affects oxygen supply and tear osmolarity, resulting in corneal swelling of $2-5 \%$ that is observed in the morning [34, 35].

Various theoretical studies have reported that to avoid the potential corneal oedema caused by scleral contact lenses, we should adjust certain characteristics of contact lenses and their fit. Michaud et al. [15] established that scleral contact lenses must be made from a material with a Dk above 150, a maximum central thickness of $250 \mu \mathrm{m}$ and an apical clearance of no more than $200 \mu \mathrm{m}$, while Compañ et al. [16] set the limits at a Dk of 124, lens thickness of $200 \mu \mathrm{m}$ and apical clearance of $150 \mu \mathrm{m}$.

Vincent et al. [19] assessed corneal thickness in healthy subjects after wearing $16.5-\mathrm{mm}$ contact lenses with a Dk of 100 for $8 \mathrm{~h}$. They found that corneal thickness increased by $1.70 \pm 0.98 \%$, that is, less than in the case of physiological oedema and although the difference was statistically significant, it was not considered clinically relevant. A study by Soeters et al. [18] in patients with keratoconus indicates, among other changes, increased corneal thickness, measured immediately after scleral contact lens removal, but again the change was not greater than that observed in physiological oedema.

Although our study was based on patients with abnormal corneas (irregular corneas), we found a non-significant increase in central corneal thickness of $2.3 \%(\mathrm{IQR}=5.6$ ). As in the study by Vicent et al. [19], the only region where corneal thickness changes were significant was in the superior cornea, where the upper eyelid covers the lens. The upper eyelid covers the superior zone of the scleral contact lens behaving as a physical barrier to atmospheric oxygen. We did not, however, find any correlation between change in corneal thickness and contact lens thickness or vault.

Unlike previous studies, we used anterior segment OCT (with the RTVue-100 system) to measure corneal thickness. According to various publications, this system has a repeatability of $1.3-1.7 \mu \mathrm{m}$ [26] or $1.2 \pm 0.8 \mu \mathrm{m}$ [27] and a correlation coefficient between successive measurements of 0.9979 [28] for normal corneas, while in abnormal corneas, repeatability has been found to be $1.0-2.6 \mu \mathrm{m}$ in cases of corneal dystrophy or scarring and $5.2 \pm 5.6 \mu \mathrm{m}$ in keratoconus [29]. Using an instrument with good measurement repeatability, it is possible to obtain values that are more comparable when monitoring corneal thickness over time. In some other work, researchers have used an Oculus Pentacam HR (Wetzlar, Germany), which has a repeatability of $4 \pm 12 \mu \mathrm{m}$ in normal corneas [36], and this greater variability might result in markedly different results.

Another factor we analysed is the variation in the contact lens-to-cornea distance, that is, the vault, which may impede the transfer of oxygen. The greater the vault, the lower partial pressure of oxygen [17]. One of the few studies to have measured apical clearance over time was conducted by Kauffman et al. [21] and analysed the behaviour of different models of scleral contact lenses (Onefit P\&A, MSD and Jupiter) in normal subjects. They measured the vault from fitting of the lenses until $8 \mathrm{~h}$ of wear and concluded that in all the contact lenses there is a decrease in central vault that stabilises after $4 \mathrm{~h}$ of wear. We found that over the months, the contact lenses (Rose $\mathrm{K} 2 \mathrm{XL}^{\mathrm{TM}}$ ) settle into the conjunctival tissue, with decreases in the central vault after 1 month of use of the contact lenses, and again at 6 and 12 months. Kauffman et al. [21] make a theoretical approximation of the vault size at 14 days, these values differ between each contact lens model. The decrease in the vault size described by these authors is greater than what we have observed after a year of Rose $\mathrm{K} 2 \mathrm{XL}^{\mathrm{TM}}$. This may suggest that a theoretical model of prediction of the behaviour of scleral contact lenses cannot be applied in patients with corneal pathology as our study. Furthermore, Kauffman et al. studies vault changes over a short period of time, whilst our patients are analysed over 1 year. So, the vault do not have to have a regular modification over time. The modification in the vault is mainly due to the settlement in the sclera where the scleral contact lens rests. The sclera can have different consistency and also the weight of the contact lens can influence. All these factors must be studied in order to establish a more accurate relationship of the expected theoretical change in the vault over time.

Finally, we should note that none of the eyes analysed had a positive result in the Limbokit test after 1 year of scleral contact lens use for a minimum of $8 \mathrm{~h}$ every day. This confirms the finding that increases in corneal thickness within the range considered physiological are not indicative of alterations in corneal physiology with Rose $\mathrm{K} 2 \mathrm{XL}^{\mathrm{TM}}$ scleral contact lens use. Although we have experience with the Limbokit test, this technique has some limitations to obtain an adequate sample from the epithelium. Both sides of each disc were placed in contact with the epithelium using sterile tweezers to obtain the highest possible number of cells.

We conclude that when the scleral contact lenses are tolerated wearing at least $8 \mathrm{~h}$ per day, the corneal thickness and corneal limbus did not modify. Therefore, it is a suitable option for the improvement of visual acuity in 
irregular corneas. Nevertheless, longer-term studies are needed to determine whether an objective test for limbal stem cell deficiency such as the Limbokit would remain negative over longer periods of time and whether the corneal thickness and vault continue to change. Similarly, we need to assess whether there are differences as a function of diseases and topographies. In addition, we should also investigate whether the changes observed are generalizable to other types of scleral contact lenses.

\section{Summary}

\section{What was known before}

- Scleral contact lens improve visual acuity after scleral contact lens removal, corneal thickness increased.

\section{What this study adds}

- No changes in corneal physiology over time with scleral contact lens, using an objective test (Limbokit).

Funding This study was funded by a Jesús de Gangoiti Barrera grant with no links between the researchers who conducted the work and the companies that supplied the materials used.

\section{Compliance with ethical standards}

Conflict of interest The authors declare that they have no conflict of interest.

Publisher's note Springer Nature remains neutral with regard to jurisdictional claims in published maps and institutional affiliations.

\section{References}

1. Bennett EB. GP annual report 2016. Contact Lens Spectr. 2016; 31:24-31.

2. Visser E-S, Van der Linden BJ, Otten HM, Van der Lelij A, Visser R. Medical applications and outcomes of bitangential scleral lenses. Optom Vis Sci. 2013;90:1078-85.

3. Romero-Jiménez M, Flores-Rodríguez P. Utility of a semi-scleral contact lens design in the management of the irregular cornea. Cont Lens Anterior Eye. 2013;36:146-50.

4. Lee JC, Chiu GB, Bach D, Babadeygy SR, Irvine J, Heur M. Functional and visual improvement with prosthetic replacement of the ocular surface ecosystem scleral lenses for irregular corneas. Cornea. 2013;32:1540-3.

5. Alipour F, Kheirkhah A, Jabarvand BM. Use of mini scleral contact lenses in moderate to severe dry eye. Cont Lens Anterior Eye. 2012;35:272-6.

6. Kalwerisky K, Davies B, Mihora L, Czyz CN, Foster JA, DeMartelaere S. Use of the Boston Ocular Surface Prosthesis in the management of severe periorbital thermal injuries: a case series of 10 patients. Ophthalmology. 2012;119:516-21.
7. Shah-Desai SD, Aslam Sa, Pullum K, Beaconsfield M, Rose GE. Scleral contact lens usage in patients with complex blepharoptosis. Ophthal Plast Reconstr Surg. 2011;27:95-8.

8. Severinsky B, Behrman S, Frucht-Pery J, Solomon A. Scleral contact lenses for visual rehabilitation after penetrating keratoplasty: long term outcomes. Contact Lens Anterior Eye. 2014;37:196-202.

9. Barnett M, Lien V, Li JY, Mannis MJ, Durbin-Johnson B. Use of scleral lenses and miniscleral lenses after penetrating keratoplasty. Eye Contact Lens Sci Clin Pr. 2016;42:185-9.

10. Kramer EG, Boshnick EL. Scleral lenses in the treatment of post-LASIK ectasia and superficial neovascularization of intrastromal corneal ring segments. Contact Lens Anterior Eye. 2015;38:298-303.

11. Schornack MM. Limbal stem cell disease: management with scleral lenses. Clin Exp Optom. 2011;94:592-4.

12. Tougeron-Brousseau B, Delcampe A, Gueudry J, Vera L. Doan S, Hoang-Xuan $\mathrm{T}$ et al. Vision-related function after scleral lens fitting in ocular complications of Stevens-Johnson syndrome and toxic epidermal necrolysis. Am J Ophthalmol. 2009;148:852-859.e2.

13. Deloss KS, Fatteh NH, Hood CT. Prosthetic replacement of the ocular surface ecosystem (PROSE) scleral device compared to keratoplasty for the treatment of corneal ectasia. Am J Ophthalmol. 2014;158:974-82.

14. Schornack MM. Scleral lenses. Eye Contact Lens Sci Clin Pr. 2015;41:3-11.

15. Michaud L, van der Worp E, Brazeau D, Warde R, Giasson CJ. Predicting estimates of oxygen transmissibility for scleral lenses. Cont Lens Anterior Eye. 2012;35:266-71.

16. Compañ V, Oliveira C, Aguilella-Arzo M, Mollá S, Peixoto-deMatos SC, Gónzalez-Méojome JM. Oxygen diffusion and edema with modern scleral rigid gas permeable contact lenses. Invest Ophthalmol Vis Sci. 2014;55:6421-9.

17. Giasson CJ, Morency J, Melillo M, Michaud L. Oxygen tension beneath scleral lenses of different clearances. Optom Vis Sci. 2017;94:466-75

18. Soeters N, Visser ES, Imhof SM, Tahzib NG. Scleral lens influence on corneal curvature and pachymetry in keratoconus patients. Contact Lens Anterior Eye. 2015;38:294-7.

19. Vincent SJ, Alonso-caneiro D, Collins MJ, Beanland A, Lam L. Hypoxic corneal changes following eight hours of scleral contact lens wear. Optom Vis Sci. 2016;93:293-9.

20. Smith GT, Mireskandari K, Pullum KW. Corneal swelling with overnight wear of scleral contact lenses. Cornea 2004;23:29-34.

21. Kauffman MJ, Gilmartin Ca, Bennett ES, Bassi CJ. A comparison of the short-term settling of three scleral lens designs. Optom Vis Sci. 2014;91:1462-6.

22. Calonge M, Duebold Y, Sáez V, Enriquez de Salamanca A, García-Vázuqez C, Corrales RM. et al. Impression cytology of the ocular surface: a review. Exp Eye Res. 2004;78:457-72.

23. Rivas L, Oroza MA, Perez E, Murube del Castillo J. Topographical distribution of ocular surface cells by the use of impression cytology. Acta Ophthalmol. 1991;69:371-6.

24. Garcia I, Etxebarria J, Boto-de-Los-Bueis A, Díaz-Valle D, Rivas L, Martínez-Soroa I. et al. Comparative study of limbal stem cell deficiency diagnosis methods: detection of MUC5AC mRNA and goblet cells in corneal epithelium. Ophthalmology 2012;119:923-9.

25. García I, Etxebarria J, Merayo-Lloves J, Torras J, Boto-de-los-Bueis A, Díaz-Valle D et al. Novel molecular diagnostic system of limbal stem cell deficiency based on MUC5AC transcript detection in corneal epithelium by PCR-reverse dot blot. Investig Ophthalmol Vis Sci. 2013;54:5643-52. 
26. Li Y, Tang M, Zhang X, Salaroli CH, Ramos JL, Huang D. Pachymetric mapping with Fourier-domain optical coherence tomography. J Cataract Refract Surg. 2010;36:826-31.

27. Wang Q, Hua Y, Savini G, Chen H, Bao F, Lin S et al. Corneal power measurement obtained by fourier-domain reproducibility, and comparison with scheimpflug and automated keratometry measurements. Cornea. 2015;34:1266-71.

28. Bayhan HA, Aslan Bayhan S, Can I. Comparison of central corneal thickness measurements with three new optical devices and a standard ultrasonic pachymeter. Int J Ophthalmol. 2014;7:302-8.

29. Wang C, Xia X, Tian B, Zhou S. Comparison of fourier-domain and time-domain optical coherence tomography in the measurement of thinnest corneal thickness in Keratoconus. J Ophthalmol 2015;2015:1-7.

30. de Luis Eguileor B, Etxebarria Ecenarro J, Santamaria Carro A, Feijoo Lera R. Irregular corneas. Eye Contact Lens Sci Clin Pr. 2016;0:1.
31. Alipour F, Jabarvand Behrouz M, Samet B. Mini-scleral lenses in the visual rehabilitation of patients after penetrating keratoplasty and deep lamellar anterior keratoplasty. Contact Lens Anterior Eye. 2015;38:54-8.

32. Kornberg DL, Dou E, Wang Y, St Clair R, Lee MN, Sood P et al. Clinical experience with PROSE fitting: significance of diagnosis and age. Eye Contact Lens. 2016;42:124-8.

33. Mishima S. Corneal thickness. Surv Ophthalmol 1968;13:57-96.

34. Mertz GW. Overnight swelling of the living human cornea. J Am Optom Assoc. 1980;51:211-4.

35. Harper CL, Boulton ME, Bennett D, Marcyniuk B, Jarvis-Evans $\mathrm{JH}$, Tullo $\mathrm{AB}$ et al. Diurnal variations in human corneal thickness. Br J Opthalmol. 1996;80:1068-72.

36. McAlinden C, Khadka J, Pesudovs K. A comprehensive evaluation of the precision (repeatability and reproducibility) of the oculus pentacam HR. Investig Ophthalmol Vis Sci. 2011; $52: 7731-7$. 